\title{
IncRNA MALAT1 overexpression promotes proliferation, migration and invasion of gastric cancer by activating the PI3K/AKT pathway
}

\author{
KONGXI ZHU ${ }^{1}$, QING REN ${ }^{1}$ and YANYING ZHAO ${ }^{2}$ \\ ${ }^{1}$ Digestive Department and ${ }^{2}$ Health Check Center, The Second Hospital of Shandong University, \\ Jinan, Shandong 250033, P.R. China
}

Received June 6, 2018; Accepted January 21, 2019

DOI: $10.3892 / \mathrm{ol} .2019 .10253$

\begin{abstract}
Long non-coding RNA (lncRNA) has been implicated in various types of human cancer. However, the role of lncRNA metastasis-associated lung adenocarcinoma transcript 1 (MALAT1) in gastric cancer remains unclear. In the present study, MALAT1 expression was significantly upregulated in gastric tumors compared with adjacent healthy tissue in patients with gastric cancer. Furthermore, MALAT1 plasma expression was higher in patients with gastric cancer compared with healthy controls and was found to have prognostic and diagnostic value independent of patients' lifestyle choices. Cell proliferation assay and Transwell migration and invasion results indicated that the overexpression of MALAT1 resulted in increased proliferation, migration and invasion of gastric cancer cells in vitro, possibly through activation of the phosphoinositide 3-kinase/protein kinase B pathway.
\end{abstract}

\section{Introduction}

Gastric cancer is the third leading cause of cancer-associated mortality world-wide (1). Numerous genetic factors have been implicated in gastric cancer (2) and a strong correlation has been identified between the activation of certain oncogenes, mutations in tumor suppressor genes and abnormal expression of key proteins and the initiation, development and progression of the disease (3). Surgical resection is the current gold standard curative therapy for gastric cancer (4). While radiation and chemotherapy have been developed, there is a requirement for safe and effective treatment strategies to decrease the mortality associated with the disease (4). It is often difficult to diagnose gastric cancer in the early stages of the disease,

Correspondence to: Dr Yanying Zhao, Health Check Center, The Second Hospital of Shandong University, 247 Beiyuan Road, Jinan, Shandong 250033, P.R. China

E-mail:vghpaubo@yeah.net

Key words: MALAT1, gastric cancer, phosphoinositide 3-kinase/ protein kinase B pathway and the majority of patients are diagnosed in the advanced stages (5). However, early diagnosis and treatment can improve patient outcomes.

The development of diseases can lead to changes in plasma composition (6) and there has been an increased interest in the identification of plasma biomarkers which can be used in the diagnosis of various human diseases including cancer $(7,8)$. Long non-coding RNAs (lncRNAs) are a group of non-coding RNAs formed by $>200$ nucleotides (9). It has been well accepted that lncRNAs participate in almost every aspect of all critical biological processes in the human body (10). Several lncRNAs have been found to serve essential roles in the development of different types of human cancer (11). Furthermore, circulating plasma lncRNAs have been used for prognostic and diagnostic evaluation of several diseases (12). IncRNA metastasis-associated lung adenocarcinoma transcript 1 (MALAT1) expression is upregulated in several malignancies $(13,14)$, emphasizing its potential role as a diagnostic indicator for these diseases. For example, overexpression of lncRNA MALAT1 is significantly correlated with poor prognosis in colorectal cancer (13) and malignant gliomas (14). Two previous studies also reported the overexpression of lncRNA MALAT1 in gastric cancer $(15,16)$. However, the function of lncRNA MALAT1 in gastric cancer has not been elucidated.

In the present study, the expression of MALAT1 in tumor tissues and adjacent healthy tissues in patients with gastric cancer was investigated. The expression of lncRNA MALAT1 in plasma of patients with gastric cancer and healthy controls was also compared. Furthermore, the diagnostic and prognostic values of plasma MALAT1 for gastric cancer were investigated. Additionally, the interactions between MALAT1 overexpression and the phosphoinositide 3-kinase $(\mathrm{PI} 3 \mathrm{~K}) /$ protein kinase B (AKT) pathway were also explored. To the best of our knowledge, the current study is the first to report the interactions between MALAT1 overexpression and the PI3K/AKT pathway in gastric cancer.

\section{Materials and methods}

Patients. A total of 64 patients with gastric cancer from The Second Hospital of Shandong University were enrolled between January 2008 and Januray 2010. All patients were 
diagnosed by imaging examinations and pathological tests. The patients included 30 males and 34 females, and the age ranged from 28 to 77 years, with a mean age of $42 \pm 13.1$ years. All patients received surgical resections and tumor tissues and healthy tissue within $2 \mathrm{~cm}$ of the tumors were collected. A control group consisting of 64 healthy people was also used. This group included 29 males and 35 females, and the age ranged from 31 to 72 years, with a mean age of $40 \pm 12.6$ years. There were no significant differences in age, sex and other basic information between the patient group and control group. All patients were followed up for 5 years after discharge. This study was approved by the Ethics Committee of The Second Hospital of Shandong University. Written informed consent was obtained from all patients prior to enrolment. All experiments were performed in accordance with The Declaration of Helsinki.

Plasma preparation. A blood sample $(100 \mathrm{ml})$ was collected from each paticipant in the morning and transferred to anticoagulant tubes. Samples were centrifuged at 1,275 x g for $10 \mathrm{~min}$ at room temperature to separate the plasma which was removed and stored at $4^{\circ} \mathrm{C}$ until use.

Cell lines and cell culture. Human gastric cancer cell lines SNU-1 (ATCC CRL-5971 ${ }^{\mathrm{TM}}$, gastric carcinoma) and AGS (ATCC CRL-1739 ${ }^{\mathrm{TM}}$, gastric adenocarcinoma) were used. Cell lines were purchased from the American Type Culture Collection (ATCC; Manassas, VA, USA). All cells were cultured with RPMI-1640 medium (cat. no. ATCC 30-2001) containing $10 \%$ fetal bovine serum (cat. no. ATCC 30-2020) at $37^{\circ} \mathrm{C}$ with $5 \% \mathrm{CO}_{2}$. Cells were harvested during the logarithmic growth phase for subsequent experiments.

Reverse transcription-quantitative polymerase chain reaction $(R T-q P C R)$. Total RNA was extracted from tumor tissues, adjacent healthy tissues, plasma and cultured SNU-1 and AGS cell lines using TRIzol ${ }^{\circledR}$ reagent (Invitrogen; Thermo Fisher Scientific, Inc., Waltham, MA, USA). RNA quality was tested by NanoDrop ${ }^{\mathrm{TM}} 2000$ Spectrophotometer (Thermo Fisher Scientific, Inc., Wilmington, DE, USA). Only RNA samples with a A260/A280 ratio between 1.8 and 2.0 were subjected to synthesize cDNA using SuperScript IV Reverse Transcriptase (Thermo Fisher Scientific, Inc.), under the following conditions: $25^{\circ} \mathrm{C}$ for $5 \mathrm{~min}, 52^{\circ} \mathrm{C}$ for $30 \mathrm{~min}$ and $80^{\circ} \mathrm{C}$ for $10 \mathrm{~min}$. qPCR was performed using SYBR ${ }^{\circledR}$ Green Real-Time PCR Master Mix (Thermo Fisher Scientific, Inc.). The following primer pairs were used for the qPCR reactions: 5'-CAGTGG GGAACTCTGACTCG-3' (forward) and 5'-GTGCCTGGT GCTCTCTTACC-3' (reverse) for MALAT1; 5'-GACCTC TATGCCAACACAGT-3' (forward) and 5'-AGTACTTGC GCTCAGGAGGA-3' (reverse) for $\beta$-actin. The thermocycling conditions used were as follows: $95^{\circ} \mathrm{C}$ for $45 \mathrm{sec}$, followed by 40 cycles of $95^{\circ} \mathrm{C}$ for $25 \mathrm{sec}$ and $60^{\circ} \mathrm{C}$ for $40 \mathrm{sec}$. Data were processed using $2^{-\Delta \Delta \mathrm{Cq}}$ method (17), and the relative expression level of MALAT1 was normalized to the endogenous control $\beta$-actin.

Establishment of MALAT1 overexpression in cell lines. Full length MALAT1 cDNA (Sangon Biotech Co., Ltd., Shanghai, China) was inserted into the pIRES2-EGFP vector (Clontech
Laboratories, Inc., Mountainview, CA, USA). Before transfection, cells $\left(5 \times 10^{4}\right.$ cells per well, $\left.10 \mathrm{ml}\right)$ from each cell line were cultured over night to reach $80-90 \%$ confluence. Lipofectamine $^{\circledR} 2000$ reagent (cat. no. 11668-019; Invitrogen; Thermo Fisher Scientific, Inc.) was used for transfection into $10^{5}$ cells in $10 \mathrm{ml}$ cell suspension with vectors at a dose of $15 \mathrm{nM}$ and incubated for $5 \mathrm{~h}$. The control group was comprised of untransfected cells, and the negative control group was comprised of cells transfected with empty vectors.

Cell counting kit-8 (CCK-8) assay. The CCK-8 assay (Sigma-Aldrich; Merck KGaA, Darmstadt, Germany) was used to evaluate cell proliferation. Briefly, $100 \mu \mathrm{l}$ of cell suspension containing $5 \times 10^{3}$ cells was transferred to each well of a 96-well plate. Cells were cultured in an incubator under normal conditions $\left(37^{\circ} \mathrm{C}, 5 \% \mathrm{CO}_{2}\right)$, and $10 \mu \mathrm{l}$ of CCK-8 solution was added into each well after 12, 24, 48, 72 and $96 \mathrm{~h}$ of cell culture. After incubation under the same conditions for another $4 \mathrm{~h}$, optical density values at $450 \mathrm{~nm}$ were measured using a microplate reader (Bio-Rad Laboratories, Inc., Hercules, CA, USA).

Cell migration and invasion assays. A total of $5 \times 10^{4}$ SNU-1 and AGS cells were transferred to the upper chambers of Transwell plates (BD Biosciences, San Jose, CA, USA) in serum-free RPMI-1640 medium. RPMI-1640 medium (Thermo Fisher Scientific, Inc.) containing 20\% fetal calf serum (Sigma-Aldrich; Merck KGaA) was used to fill the lower chamber. Following incubation for $24 \mathrm{~h}$ at $37^{\circ} \mathrm{C}$, the migrated cells were stained with $0.5 \%$ crystal violet (Sigma-Aldrich; Merck KGaA) for $15 \mathrm{~min}$. Five visual fields were randomly selected and stained cells were then counted under an optical microscope (Olympus Corporation, Tokyo, Japan). In the cell invasion assay, the upper chamber was pre-coated with Matrigel (cat. no. 356234, EMD Millipore, Billerica, MA, USA) overnight at room temperature.

Western blotting. Total protein was extracted from cultured cells of SNU-1 and AGS cell lines using cell lysis buffer (cat. no. 9803; Cell Signaling Technology, Inc., Danvers, MA, USA). Total protein was quantified using a bicinchoninic acid assay and $50 \mu \mathrm{g}$ protein/lane was separated via SDS-PAGE on a $10 \%$ gel. The separated proteins were subsequently transferred onto a polyvinylidene difluoride membrane which was then blocked for $1 \mathrm{~h}$ at room temperature with 5\% skimmed milk (Sigma-Aldrich; Merck KGaA). Membranes were incubated with primary antibodies against PI3K (1:2,000; cat. no. ab5451; Abcam, Cambridge, UK), p-PI3K (1:2,000; cat. no. ab182651; Abcam), AKT (1:2,000; cat. no. ab126811; Abcam), p-AKT (1:2,000; cat. no. ab18206; Abcam) and GAPDH (1:1,000, cat. no. ab9485; Abcam) overnight at $4^{\circ} \mathrm{C}$. After washing, membranes were incubated with a horseradish peroxidase-labeled secondary antibody (1:1,000; cat. no. MBS435036; MyBioSource Inc., San Diego, CA, USA) for $2 \mathrm{~h}$ at room temperature. After washing, protein bands were detected using ECL ${ }^{\text {TM }}$ Detection Reagents (Sigma-Aldrich; Merck KGaA). This experiment was performed in triplicate and protein expression was quantified using ImageJ 1.46 software (National Institutes of Health, Bethesda, MD, USA) with GAPDH as the loading control. 


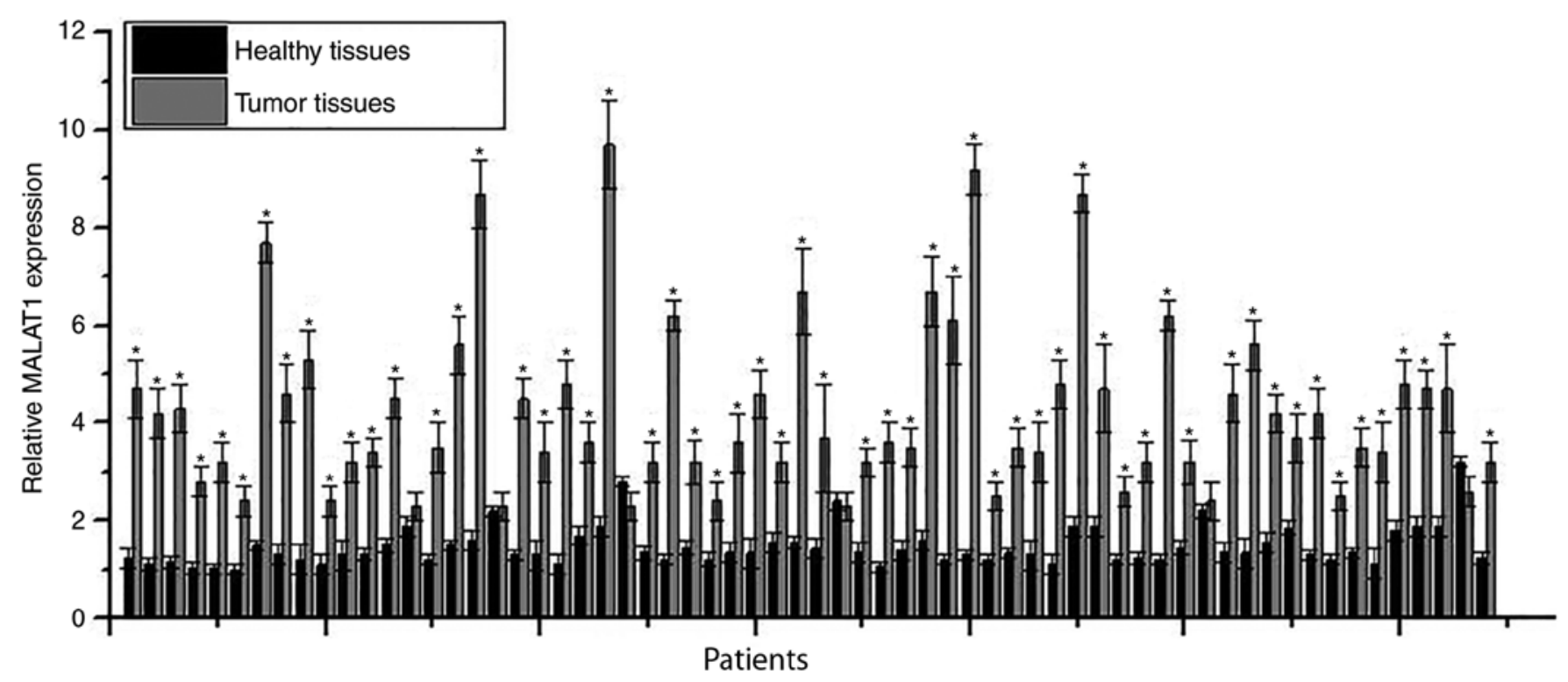

Figure 1. Relative expression level of MALAT1 in tumor tissues and adjacent tissues of patients with gastric cancer. "P $<0.05$ vs. healthy tissue. MALAT1, metastasis-associated lung adenocarcinoma transcript 1 .

Statistical analysis. SPSS software (version 19.0; IBM Corp., Armonk, NY, USA) was used. Normal distribution data were expressed as mean \pm standard deviation, and comparisons between two groups were performed by t-test. Comparisons among multiple groups were performed by one way analysis of variance, followed by Tukey test. Non-normal distribution data were analyzed by non-parametric Mann-Whitney $\mathrm{U}$ test. Effects of patients' basic information on expression of MALAT1 cancer were analyzed by Chi-square test and Bonferroni's correction. Receiver operating characteristic (ROC) curve analysis was performed to evaluate the diagnostic value of plasma levels of MALAT1 for gastric cancer. Patients were divided into high MALAT1 expression group and low MALAT1 expression group according to the median plasma level of MALAT1 $(n=32)$. Survival curves were plotted using Kaplan-Meier method and compared by log-rank test. Pearson correlation analysis was performed to analyze the correlation between plasma levels of MALAT1 with the expression levels of MALAT1 in tumor tissues and adjacent healthy tissues. A P $<0.05$ was considered to indicate a statistically significant difference.

\section{Results}

MALAT1 expression is upregulated in tumor tissues compared with adjacent healthy tissues. The expression of MALAT1 in tumor tissues and adjacent healthy tissues in all 64 patients with gastric cancer was studied. Expression levels of MALAT1 were significanly higher in tumor tissues than in adjacent healthy tissues in 59 out of 64 patients with gastric cancer (Fig. 1). This suggested that upregulated expression of MALAT1 is likely involved in the development of gastric cancer.

Plasma MALAT1 expression has diagnostic and prognostic value in gastric cancer. The expression of MALAT1 in plasma of patients with gastric cancer was significantly higher than in healthy controls $(\mathrm{P}=0.0154$; Fig. 2A). In addition, Pearson correlation analysis revealed that plasma levels of MALAT1 were positively correlated with the expression levels of MALAT1 in tumor tissues ( $\mathrm{r}=0.8745$; $\mathrm{R}$ square $=0.7647$; $\mathrm{P}<0.0001$; Fig. $2 \mathrm{~B}$ ), but not with the expression levels of MALAT1 in adjacent healthy tissues $(\mathrm{r}=0.1615 ; \mathrm{R}$ square $=0.02608 ; \mathrm{P}=0.3774$; Fig. 2C $)$. ROC analysis was performed to analyze the diagnostic value of plasma MALAT1 for gastric cancer. The area under the curve was 0.8984 with a $95 \%$ confidence interval of $0.8465-0.9504$ $(\mathrm{P}<0.0001$; Fig. 2D), indicating that plasma MALAT1 can be used to accurately predict gastric cancer. Patients were divided into high MALAT1 expression group and low MALAT1 expression group according to the median plasma level of MALAT1 $(n=32)$. Survival curves were estimated using the Kaplan-Meier method and were used to evaluate the prognostic value of plasma MALAT1 for gastric cancer. Patients with high plasma level of MALAT1 had significantly shorter survival compared with patients with a low plasma level of MALAT1 $(\mathrm{P}<0.0214$; Fig. 2D), indicating that the plasma level of MALAT1 may serve as diagnostic and prognosic indicator of gastric cancer.

Association between MALAT1 expression level and basic information of gastric cancer patients. It has been reported that the expression of IncRNAs can be affected by lifestyle choices including vegetarianism, smoking and drinking (14). This may influence the diagnostic and prognostic values of specific IncRNA for certain diseases. In view of this, effects of patients' basic information on expression of MALAT1 in gastric cancer were analyzed by Chi-square test. Data were subjected to Bonferroni's correction. MALAT1 expression levels in plasma (Table I) and tumor tissues (Table II) were not significantly affected by sex, age, smoking, drinking and vegetarianism. However, expression levels of MALAT1 were significantly higher in patients with tumor metastasis than in patients without tumor metastasis. This data suggested 
Table I. Association between the plasma level of metastasis-associated lung adenocarcinoma transcript 1 and basic information of patients with gastric cancer.

\begin{tabular}{|c|c|c|c|c|c|}
\hline Characteristic & Group & Number of cases & High-expression & Low-expression & P-value \\
\hline \multirow[t]{2}{*}{ Sex } & Male & 30 & 17 & 13 & 0.31 \\
\hline & Female & 34 & 15 & 19 & \\
\hline \multirow[t]{2}{*}{ Age } & $>40$ (years) & 35 & 18 & 17 & 0.80 \\
\hline & $<40$ (years) & 29 & 14 & 15 & \\
\hline \multirow[t]{2}{*}{ Vegetarianism } & Yes & 12 & 5 & 7 & 0.52 \\
\hline & No & 52 & 27 & 25 & \\
\hline \multirow[t]{2}{*}{ Smoking } & Yes & 33 & 17 & 16 & 0.80 \\
\hline & No & 31 & 15 & 16 & \\
\hline \multirow[t]{2}{*}{ Drinking } & Yes & 35 & 18 & 17 & 0.80 \\
\hline & No & 29 & 14 & 15 & \\
\hline \multirow{2}{*}{ Metastasis } & Yes & 37 & 30 & 7 & $<0.01$ \\
\hline & No & 27 & 2 & 25 & \\
\hline
\end{tabular}
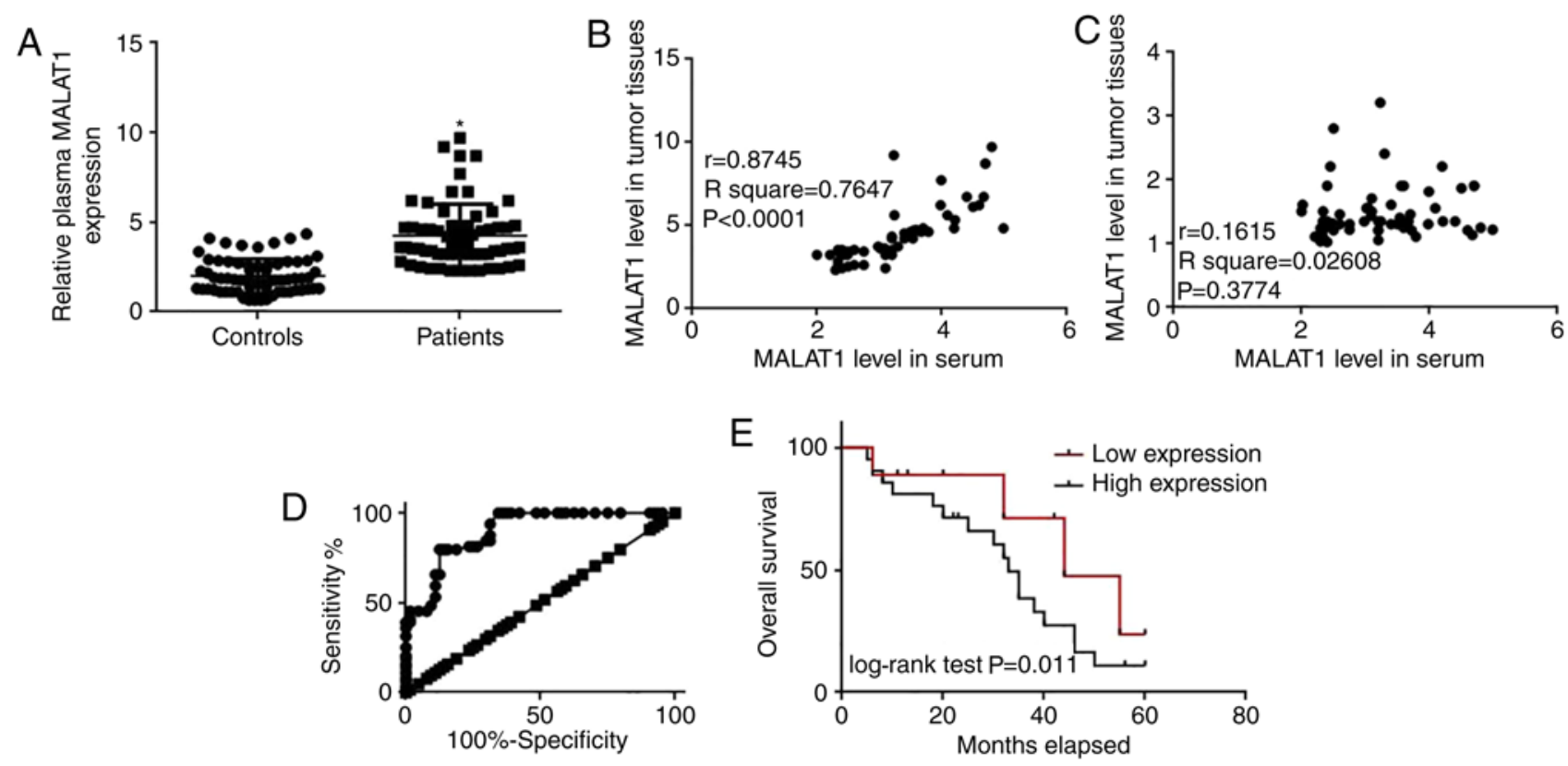

Figure 2. Plasma MALAT1 expression in patients with gastric cancer and healthy controls and its diagnostic and prognostic values. (A) Plasma MALAT1 expression in patients with gastric cancer and healthy controls. (B) Plasma levels of MALAT1 were significantly positively correlated with the expression levels of MALAT1 in tumor tissues. (C) Plasma levels of MALAT1 were not significantly correlated with the expression levels of MALAT1 in adjacent healthy tissues. (D) Receiver operating characteristic curve analysis to evaluate the diagnostic value of plasma MALAT1 for gastric cancer. (E) Survival curve analysis to evaluate the prognostic value of plasma MALAT1 for gastric cancer. ${ }^{*} \mathrm{P}<0.05$ vs. healthy control. MALAT1, metastasis-associated lung adenocarcinoma transcript 1 .

that plasma MALAT1 levels can accurately diagnose gastric cancer in patients with different lifestyle choices as well as provide an indication of the severity of the disease.

Effects of MALAT1 overexpression on the proliferation, migration and invasion of the gastric cancer cell lines $S N U-1$ and $A G S$. Gastric carcinoma and gastric adenocarcinoma are two major types of gastric cancer (1). Therefore, both the gastric carcinoma cell line SNU-1 and gastric adenocarcinoma cell line AGS were included in the current study. Effects of MALAT1 overexpression on the proliferation, migration and invasion of these two gastric cancer cell lines were assessed by the CCK-8 assay, Transwell cell migration assay and Transwell cell invasion assay. After transfection, MALAT1 overexpression was achieved in cells of both cell lines (Fig. 3A). MALAT1 overexpression significantly increased proliferation compared with control untransfected cells ( $\mathrm{P}<0.05$; Fig. 3B). Furthermore, the migratory (Fig. 3C) and invasive (Fig. 3D) abilities of both SUNP-1 and AGS cell lines were significantly increased with MALAT1 overexpression $(\mathrm{P}<0.05)$, compared with the NC group. These results indicated that MALAT1 expression can increase proliferation, migration and invasion 
Table II. Association between the expression level of metastasis-associated lung adenocarcinoma transcript 1 in tumor tissues and basic information of patients with gastric cancer.

\begin{tabular}{|c|c|c|c|c|c|}
\hline Characteristic & Group & Number of cases & High-expression & Low-expression & P-value \\
\hline \multirow[t]{2}{*}{ Sex } & Male & 30 & 18 & 12 & 0.13 \\
\hline & Female & 34 & 14 & 20 & \\
\hline \multirow[t]{2}{*}{ Age } & $>40$ (years) & 35 & 19 & 16 & 0.45 \\
\hline & $<40$ (years) & 29 & 13 & 16 & \\
\hline \multirow[t]{2}{*}{ Vegetarianism } & Yes & 12 & 5 & 7 & 0.52 \\
\hline & No & 52 & 27 & 25 & \\
\hline \multirow[t]{2}{*}{ Smoking } & Yes & 33 & 18 & 15 & 0.45 \\
\hline & No & 31 & 14 & 17 & \\
\hline \multirow[t]{2}{*}{ Drinking } & Yes & 35 & 15 & 20 & 0.21 \\
\hline & No & 29 & 17 & 12 & \\
\hline \multirow[t]{2}{*}{ Metastasis } & Yes & 37 & 29 & 8 & $<0.01$ \\
\hline & No & 27 & 3 & 24 & \\
\hline
\end{tabular}

of both gastric carcinoma cells and gastric adenocarcinoma cells.

Effects of MALAT1 overexpression on the PI3K/AKT pathway. Effects of MALAT1 overexpression on the PI3K/AKT pathway were investigated in the current study. MALAT1 overexpression had no effect on the expression levels of total PI3K and AKT in both the gastric carcinoma cell line SNU-1 (Fig. 4A) and gastric adenocarcinoma cell line AGS (Fig. 4B). However, compared with the control cells and negative control cells, levels of p-PI3K and p-AKT were significantly increased in cells with MALAT1 overexpression (Fig. 4). This suggested that MALAT1 can promote the progression of gastric cancer by increasing the phosporylation level of PI3K and AKT, but not by increasing their total expression levels.

\section{Discussion}

The results obtained in the current study confirm previous reports of the overexpression of lncRNA MALAT1 in gastric cancer $(15,16)$. Additionally, the present study explored the use of plasma MALAT1 as a diagnostic and prognostic marker in the disease. The results obtained provided evidence for the interactions between IncRNA MALAT1 and the PI3K/AKT pathway in gastric cancer, in addition to plasma MALAT1 serving as potential prognosic and diagnostic biomarker for gastric cancer.

The development of gastric cancer is a complex process and various internal and external factors are involved (3). During the past several decades, genetic factors have been demonstrated to serve pivotal roles in almost every aspect of gastric cancer (3). Despite an increased understanding of the nature of gastric cancer, the underlying pathogenesis of this disease has not been fully elucidated, leading to ineffective treatment and poor diagnosis of the disease (1). Development of gastric cancer is accompanied by changes in the expression levels of certain lncRNAs, and the abnormal expression of these lncRNAs serves different roles in the progression of the disease $(18,19)$. IncRNA H19, imprinted maternally expressed transcript (H19) a well-studied lncRNA with oncogenic functions in various types of human cancer, is overexpressed in gastric cancer, and upregulated H19 expression has been found to promote carcinogenesis and metastasis (18). By contrast, lncRNA HMlincRNA717 (gastric cancer associated transcript 2) is downregulated in patients with gastric cancer, indicating its role as a tumor suppressor (19). IncRNA MALAT1 expression has been reported to be upregulated in several types of malignancy including colorectal cancer (13), glioma (14) and gastric cancer $(15,16)$. Consistent with previous studies, the expression level of MALAT1 was significanly higher in tumor tissues compared with adjacent healthy tissues in 59 out of 64 patients with gastric cancer in the current study. Plasma MALAT1 was also significanly higher in gastric cancer patients compared with healthy controls, indicating that MALAT1 overexpression is involved in the development of gastric cancer.

The results of the current study indicated that MALAT1 may be an effective diagnostic marker for gastric cancer. In addition, patients with gastric cancer who had a higher expression level of plasma MALAT1 exhibited significantly shorter survival compared with patients with lower expression level of plasma MALAT1, indicating that plasma MALAT1 is a promising prognostic marker for gastric cancer. However, lncRNA expression can be induced or regulated by an individual's lifestyle. For example, expression of lncRNA SCAL1 can be induced by smoking (20). Therefore, the effects of patients' lifestyle choices on the expression level of plasma MALAT1 were investigated in the current study. Sex, age and lifestyle choices including smoking, drinking and vegetarianism showed no significant effects on the expression level of MALAT1. However, expression levels of MALAT1 were significantly increased in patients with tumor metastasis compared with patients without tumor metastasis.

lncRNA MALAT1 serves different roles in different types of human cancers. In the study of colorectal cancer, Yang et al (21) found that MALAT1 promoted tumor development and progression by increasing the proliferation, migration and invasion abilities of tumor cells, indicating its 
SUN-1
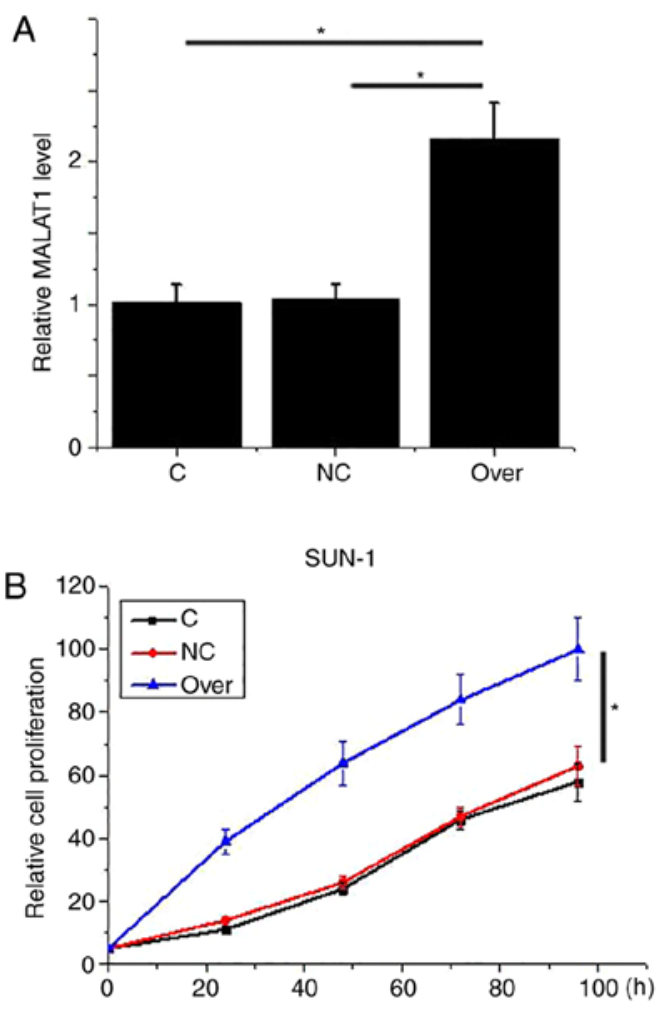

SUN-1

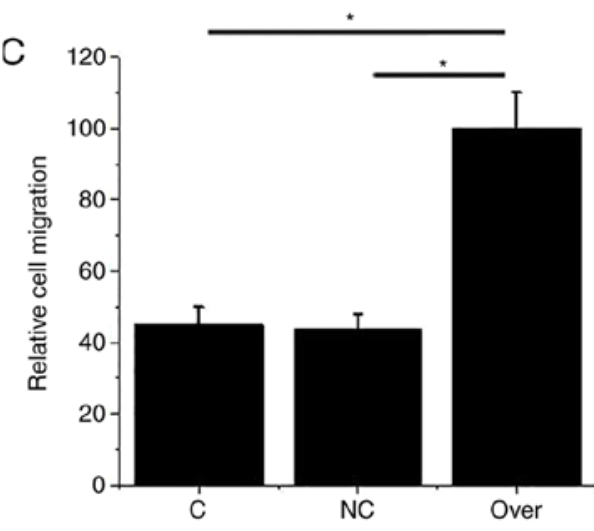

SUN-1

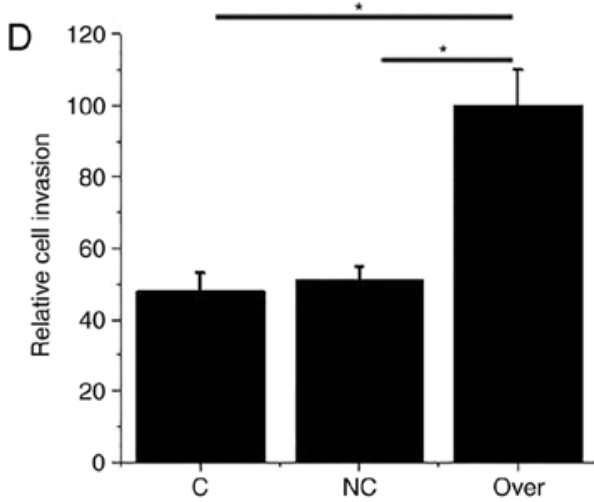

AGS

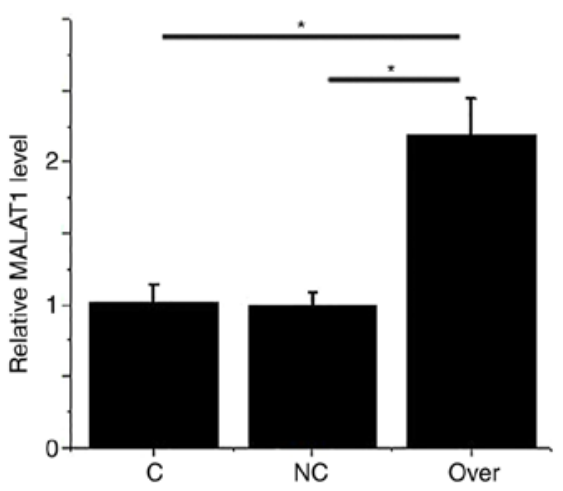

AGS

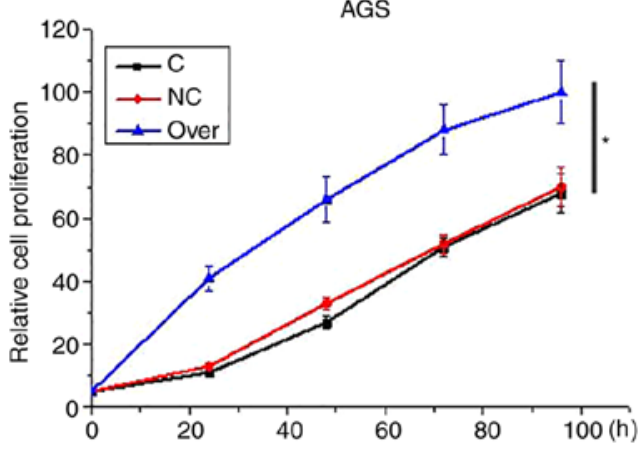

AGS

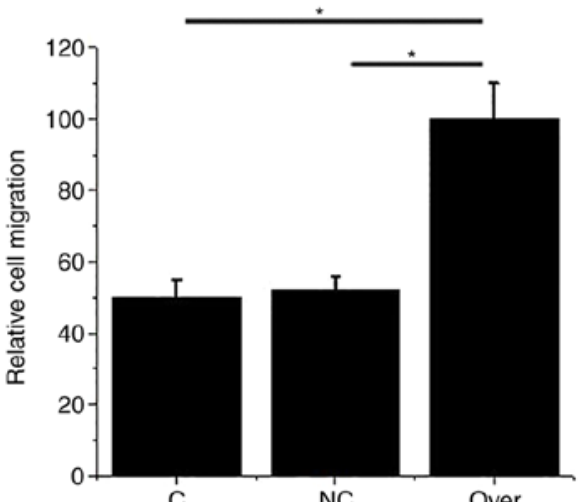

C

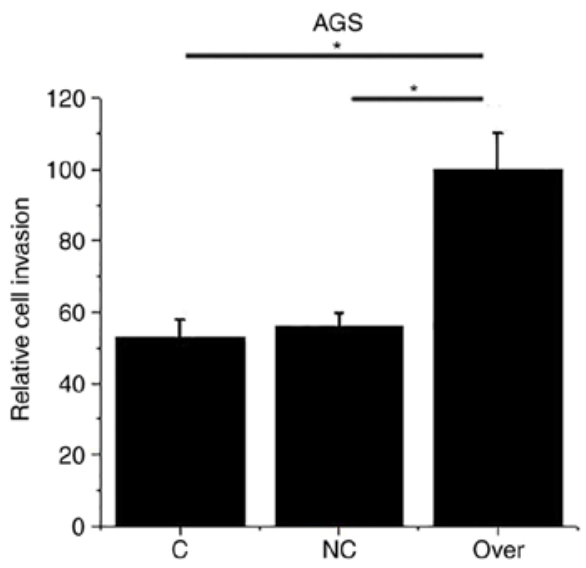

Figure 3. Effects of MALAT1 overexpression on proliferation, migration and invasion of gastric carcinoma cell line SNU-1 and gastric adenocarcinoma cell line AGS. (A) Following transfection, MALAT1 overexpression was achieved in cells of both cell lines. (B) Effects of MALAT1 overexpression on proliferation of gastric carcinoma cell line SNU-1 and gastric adenocarcinoma cell line AGS. (C) Effects of MALAT1 overexpression on migration of gastric carcinoma cell line SNU-1 and gastric adenocarcinoma cell line AGS. (D) Effects of MALAT1 overexpression on invasion of gastric carcinoma cell line SNU-1 and gastric adenocarcinoma cell line AGS. "P<0.05 vs. controls. MALAT1, metastasis-associated lung adenocarcinoma transcript 1; over, overexpression of MALAT1; NC, negative control; C, control. 
A
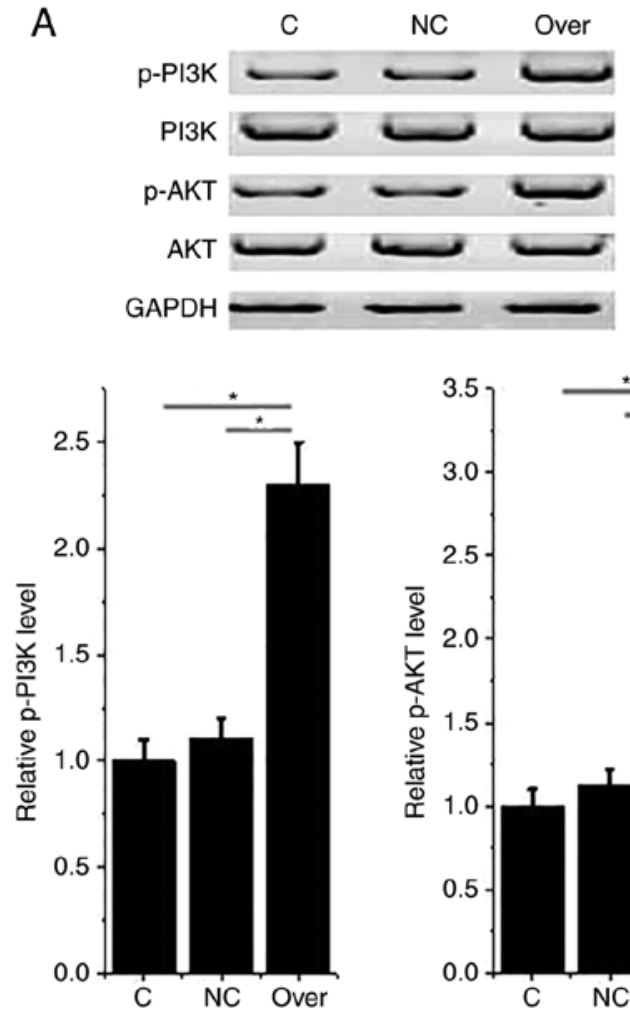
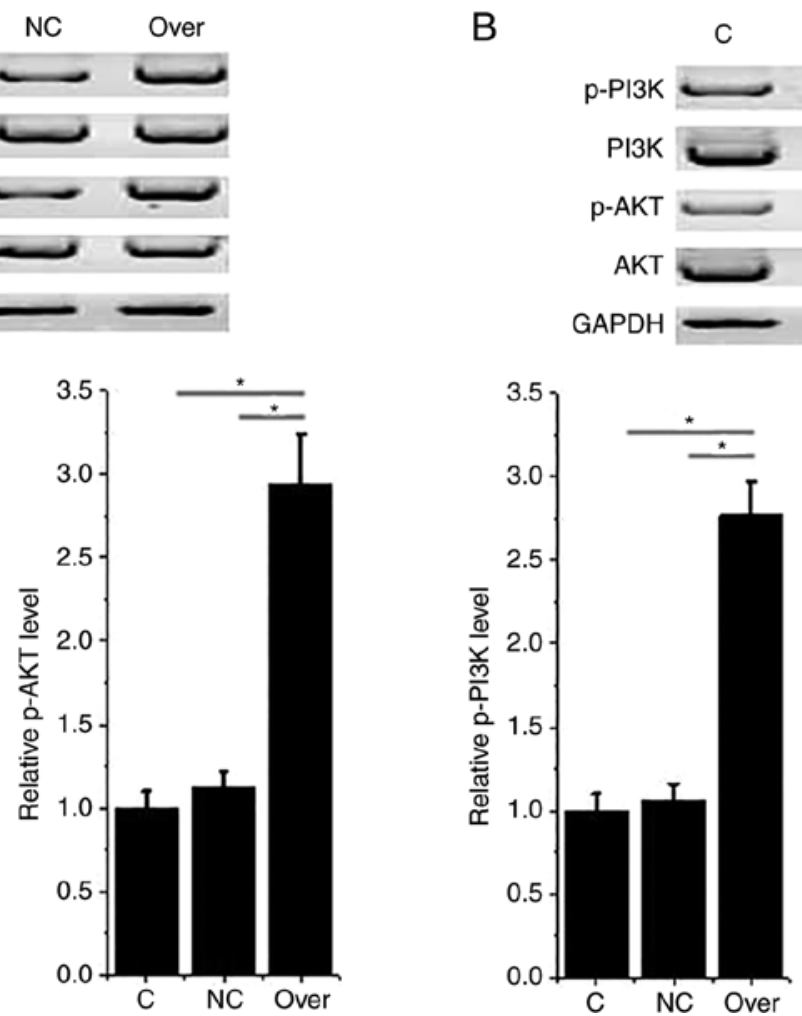

NC Over
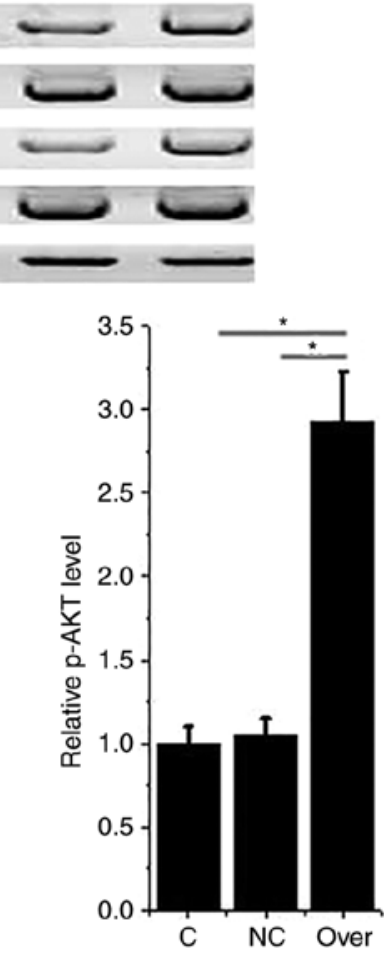

Figure 4. Effects of MALAT1 overexpression on the PI3K/AKT pathway. (A) Effects of MALAT1 overexpression on the PI3K/AKT pathway in gastric carcinoma cell line SNU-1. (B) Effects of MALAT1 overexpression on the PI3K/AKT pathway in gastric adenocarcinoma cell line AGS. *P<0.05 vs. controls. MALAT1, metastasis-associated lung adenocarcinoma transcript 1; PI3K, phosphoinositide 3-kinase; AKT, protein kinase B; over, overexpression of MALAT1; NC, negative control; C, control; p-, phosphorylated.

oncogenic role in this disease. By contrast, downregulation of IncRNA MALAT1 was found to be responsible for the induction of epithelial-to-mesenchymal transition in breast cancer (19), suggesting that lncRNA MALAT1 can also serve a tumor suppressor role in breast cancer. A previous study has reported that MALAT1 promoted the proliferation of gastric cancer cells (15). In the present study, MALAT1 overexpression significantly promoted the proliferation, migration and invasion of both the gastric carcinoma cell line SNU-1 and the gastric adenocarcinoma cell line AGS, indicating that MALAT1 serves an oncogenic role in gastric cancer by promoting cancer cell proliferation, migration and invasion. It has been previously reported that lncRNA MALAT1 interacts with the PI3K-AKT pathway (22). In the current study, MALAT1 overexpression activated the PI3K-AKT pathway in both the gastric carcinoma cell line SNU-1 and the gastric adenocarcinoma cell line AGS by promoting the phosphorylation of PI3K and AKT. This suggests that lncRNA MALAT1 can promote the development and progression of gastric cancer by activating the PI3K/AKT pathway.

While the current study highlights the importance of IncRNA MALAT1 in gastric cancer, it has certain limitations. Factors such as working habits, which can have a significant effect on cancer development (23), were not included. Such factors will be included in future studies. Although representative, the present study was limited by a relatively small sample size. Further studies with larger sample sizes are required to confirm the data obtained in this study. The current study revealed a potential interaction between MALAT1 and the
PI3K-AKT pathway in gastric cancer, which is a novel finding. However, whether the interaction between MALAT1 and $\mathrm{PI} 3 \mathrm{~K}-\mathrm{AKT}$ is direct or indirect is unknown. Our future studies will aim to establish the pathways involved.

The current study failed to analyze the correlations between expression levels of lncRNA MALAT1 with PI3K and AKT due to the limited resources. Future studies will attempt to include this analysis. Although the current data partially overlap with the data presented in a previous study (16), it revealed a new signaling pathway involvled in the MALAT1-mediated behaviors of gastric cancer cells. It is known that lncRNA MALAT1 participate in cancer biology by interacting with different pathways, including histone-lysine N-methyltransferase EZH (24) and extracellular-signal-regulated kinase/mitogen activated protein kinase (25) pathways. However, those pathways failed to repond to the overexpression of MALAT in gastric cancer cells (data not shown), indicating that MALAT1 may participate in different types of cancer by interacting with different pathways.

In conclusion, MALAT1 expression was significanly upregulated in tumor tissues compared to adjacent healthy tissues in the majority of the patients with gastric cancer studied. MALAT1 plasma levels were significantly increased in patients with gastric cancer compared with healthy controls. MALAT1 overexpression significantly promoted the proliferation, migration and invasion of gastric cancer cells and activated the PI3K/AKT pathway. Therefore, MALAT1 overexpression may promote the proliferation, migration and invasion of gastric cancer cells by activating the PI3K/AKT 
pathway and may guide the development of novel strategies for the treatment of gastric cancer.

\section{Acknowledgements}

Not applicable.

\section{Funding}

No funding was recevied.

\section{Availability of data and materials}

The datasets used and/or analyzed during the current study are available from the corresponding author on reasonable request.

\section{Authors' contributions}

YZ designed experiment. KZ performed experiment. QR analyzed data. YZ interpreted the data and drafted the manuscript. All authors reviewed and approved the manuscript.

\section{Ethics approval and consent to participate}

This study was approved by the Ethics Committee of The Second Hospital of Shandong University. All experiments were performed in accordance with The Declaration of Helsinki. Written informed consent was obtained from all patients prior to enrolment.

\section{Patient consent for publication}

All participants gave consent to the publication of the present study.

\section{Competing interests}

The authors declare that they have no competing interests.

\section{References}

1. Rugge M, Fassan M and Graham DY: Epidemiology of gastric cancer. In: Gastric Cancer. Strong VE (ed.). Springer International Publishing, Cham, pp23-34, 2015.

2. Mocellin S, Verdi D, Pooley KA and Nitti D: Genetic variation and gastric cancer risk: A field synopsis and meta-analysis. Gut 64: 1209-1219, 2015.

3. Oliveira C, Pinheiro H, Figueiredo J, Seruca R and Carneiro F: Familial gastric cancer: Genetic susceptibility, pathology, and implications for management. Lancet Oncol 16: e60-e70, 2015.

4. Orditura M, Galizia G, Sforza V, Gambardella V, Fabozzi A, Laterza MM,Andreozzi F, Ventriglia J, Savastano B,Mabilia A, et al: Treatment of gastric cancer. World J Gastroenterol 20: 1635-1649, 2014.

5. Choi KS, Jun JK, Suh M, Park B, Noh DK, Song SH, Jung KW, Lee HY, Choi IJ and Park EC: Effect of endoscopy screening on stage at gastric cancer diagnosis: Results of the National Cancer Screening Programme in Korea. Br J Cancer 112: 608-612, 2015.

6. Henriksen K, O'Bryant SE, Hampel H, Trojanowski JQ, Montine TJ, Jeromin A, Blennow K, Lönneborg A, Wyss-Coray T, Soares $\mathrm{H}$, et al: The future of blood-based biomarkers for Alzheimer's disease. Alzheimers Dement 10: 115-131, 2014.
7. Hori SS, Lutz AM, Paulmurugan R and Gambhir SS: Correlation of plasma biomarker levels with early-stage tumor viability in an orthotopic ovarian cancer mouse model. Clin Cancer Res (Suppl.): 873, 2014.

8. Takahari D, Wakatsuki T, Mashima T, Ichimura T, Chin K, Ogura M, Nakayama I, Matsushima T, Osumi H, Ozaka M, et al: Plasma biomarker analysis of ramucirumab in Japanese patients with advanced gastric cancer: Preliminary results. J Clin Oncol 36 (Suppl.): 81, 2018.

9. Perkel JM: Visiting 'noncodarnia'. Biotechniques 54: 301, 303-304, 2013.

10. Esteller M: Non-coding RNAs in human disease. Nat Rev Genet 12: 861-874, 2011.

11. Prensner JR and Chinnaiyan AM: The emergence of lncRNAs in cancer biology. Cancer Discov 1: 391-407, 2011.

12. Zhang K, Luo Z, Zhang Y, Zhang L, Wu L, Liu L, Yang J, Song X and Liu J: Circulating lncRNA H19 in plasma as a novel biomarker for breast cancer. Cancer Biomark 17: 187-194, 2016.

13. Zheng HT, Shi DB, Wang YW, Li XX, Xu Y, Tripathi P, Gu WL, Cai GX and Cai SJ: High expression of lncRNA MALAT1 suggests a biomarker of poor prognosis in colorectal cancer. Int J Clin Exp Pathol 7: 3174, 2014.

14. Ma KX, Wang HJ, Li XR, Li T, Su G, Yang P and Wu JW: Long noncoding RNA MALAT1 associates with the malignant status and poor prognosis in glioma. Tumor Biol 36: 3355-3359, 2015.

15. Wang J, Su L, Chen X, Li P, Cai Q, Yu B, Liu B, Wu W and Zhu Z: MALAT1 promotes cell proliferation in gastric cancer by recruiting SF2/ASF. Biomed Pharmacother 68: 557-564, 2014.

16. Xia H, Chen Q, Chen Y, Ge X, Leng W, Tang Q, Ren M, Chen L, Yuan D, Zhang Y, et al: The lncRNA MALAT1 is a novel biomarker for gastric cancer metastasis. Oncotarget 7: 56209-56218, 2016.

17. Livak KJ and Schmittgen TD: Analysis of relative gene expression data using real-time quantitative PCR and the 2(-Delta Delta C(T) method. Methods 25: 402-408, 2001.

18. Li H, Yu B, Li J, Su L, Yan M, Zhu Z and Liu B: Overexpression of lncRNA H19 enhances carcinogenesis and metastasis of gastric cancer. Oncotarget 5: 2318-2329, 2014.

19. Shao Y, Chen H, Jiang X, Chen S, Li P, Ye M, Li Q, Sun W and Guo J: Low expression of lncRNA-HMlincRNA717 in human gastric cancer and its clinical significances. Tumor Biol 35: 9591-9595, 2014

20. Thai P, Statt S, Chen $\mathrm{CH}$, Liang E, Campbell $\mathrm{C}$ and Wu R: Characterization of a novel long noncoding RNA, SCAL1, induced by cigarette smoke and elevated in lung cancer cell lines. Am J Respir Cell Mol Biol 49: 204-211, 2013.

21. Yang MH, Hu ZY, Xu C, Xie LY, Wang XY, Chen SY and Li ZG: MALAT1 promotes colorectal cancer cell proliferation/migration/invasion via PRKA kinase anchor protein 9. Biochim Biophys Acta 1852: 166-174, 2015.

22. Xu S, Sui S, Zhang J, Bai N, Shi Q, Zhang G, Gao S, You Z, Zhan C, Liu F and Pang D: Downregulation of long noncoding RNA MALAT1 induces epithelial-to-mesenchymal transition via the PI3K-AKT pathway in breast cancer. Int J Clin Exp Pathol 8: 4881-4891, 2015.

23. Kirshners A, Polaka I and Aleksejeva L: Gastric cancer risk analysis in unhealthy habits data with classification algorithms. Inform Technol Manag Sci 18: 97-102, 2015.

24. Hirata H, Hinoda Y, Shahryari V, Deng G, Nakajima K, Tabatabai ZL, Ishii $\mathrm{N}$ and Dahiya R: Long noncoding RNA MALAT1 promotes aggressive renal cell carcinoma through Ezh2 and interacts with miR-205. Cancer Res 175: 1322-1331, 2015.

25. Wu XS, Wang XA, Wu WG, Hu YP, Li ML, Ding Q, Weng H, Shu YJ, Liu TY, Jiang L, et al: MALAT1 promotes the proliferation and metastasis of gallbladder cancer cells by activating the ERK/MAPK pathway. Cancer Biol Ther 15: 806-814, 2014.

This work is licensed under a Creative Commons Attribution-NonCommercial-NoDerivatives 4.0 International (CC BY-NC-ND 4.0) License. 